\title{
Características Biométricas de los Ligamentos Meniscofemorales en Individuos Chilenos
}

\author{
Biometrical Characteristics of Meniscofemoral Ligaments in Chilean Individuals
}

Villarroel, G.***** \& Olave, E. ${ }^{* * * * * * *}$

VILLARROEL, G. \& OLAVE, E. Características biométricas de los ligamentos meniscofemorales en individuos chilenos. Int. J. Morphol., 34(3):860-865, 2016.

RESUMEN: El conocimiento de los ligamentos meniscofemorales anterior y posterior (LMFA y LMFP) es de gran importancia para la anatomía y clínica quirúrgica. Con el propósito de efectuar un análisis de su frecuencia y biometría en individuos Chilenos, se disecaron 30 rodillas, de cadáveres de individuos adultos, de sexo no conocido, fijadas en formaldehído al $10 \%$ y pertenecientes a los Laboratorios de Anatomía, de la Facultad de Medicina de la Universidad Católica del Maule, Chile. Utilizando un caliper digital se registraron medidas de longitud, ancho y espesor de los mismos a diferentes niveles. Todas las muestras fueron fotografiadas. En relación

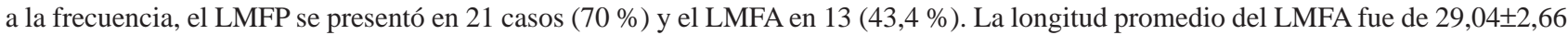

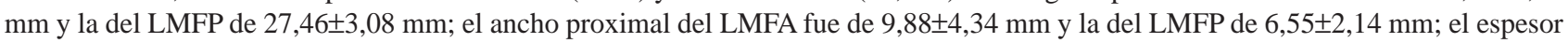

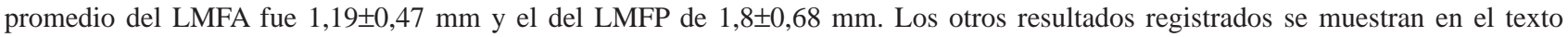
correspondiente. Los resultados obtenidos son un aporte al conocimiento anatómico de los ligamentos de la articulación de la rodilla en nuestra población.

PALABRAS CLAVE: Anatomía; Rodilla; Ligamento meniscofemoral anterior; Ligamento meniscofemoral posterior; Ligamento cruzado posterior.

\section{INTRODUCCIÓN}

El ligamento cruzado posterior (LCP) está reforzado por dos ligamentos que unen el cuerno posterior del menisco lateral a la cara externa del cóndilo medial del fémur en la incisura intercondílea, denominándose a estas estructuras ligamentos meniscofemorales (LMF). El ligamento meniscofemoral anterior (LMFA) se ubica ventral al LCP, conociéndose a éste en la literatura médica como ligamento de Humphrey. El ligamento meniscofemoral posterior (LMFP) transcurre dorsal al LCP y se conoce como ligamento de Wrisberg (Brantigan \& Voshell, 1946). Poirier \& Charpy (1899) dieron a estos ligamentos el nombre de "tercer ligamento cruzado".

En la literatura, anatómica autores clásicos como Testut \& Jacob (1961) y García-Porrero \& Hurlé (2005) señalaron que desde la extremidad posterior del menisco lateral, se origina un conjunto de fibras que se inserta en el cóndilo medial del fémur. Poirier \& Charpy; Orts Llorca (1963); Bouchet \& Cuilleret (1984); Latarjet et al. (2011) y Moore et al. (2010) añadieron que el ligamento meniscofemoral asciende aplicado a la cara posterior del LCP.

Testut \& Latarjet (1969); Chatain \& Bustamante (1986); Williams (2001) y Rouvière et al. (2005) indicaron que desde el extremo posterior del menisco lateral se desprende una banda fibrosa hacia el cóndilo medial del fémur, que puede pasar por delante o por detrás del LCP y constituye según su trayecto el ligamento meniscofemoral anterior o posterior. Testut \& Latarjet describieron a los LMF como fascículos accesorios, los cuales comúnmente se confunden en el curso de su trayecto con los fascículos correspondientes del LCP, sin embargo en ciertos casos conserva su independencia hasta su inserción femoral. El fascículo accesorio posterior puede reemplazar al fascículo accesorio anterior, pero en la mayoría de los casos se encuentran ambos. Además Rouvière et al. mencionaron que la disposición anterior del ligamento en relación al LCP es menos frecuente que su ubicación posterior, pero en ciertas ocasiones se encuentran ambos ligamentos.

\footnotetext{
* Departamento de Salud, Universidad de Los Lagos, Osorno, Chile.

** Programa de Magister en Ciencias, mención Morfología, Universidad de La Frontera, Temuco, Chile.

***Facultad de Medicina, Universidad de La Frontera, Temuco, Chile.
} 
En trabajos previos se han reportado la variabilidad de estas estructuras con respecto a la frecuencia (Heller \& Langman, 1964; Kohn \& Moreno, 1995; Wan \& Felle, 1995; Ranalleta et al., 2004), otros investigadores además han informado diferencias de longitud (Gupte et al., 2002a; Han et al., 2012) y también de anchura (Candiolo \& Guatero, 1959; Poynton et al., 1997; Erbagci et al., 2002), entre otros parámetros.

Con el propósito de complementar el conocimiento anatómico y biométrico de estos ligamentos, realizamos un estudio de los mismos, esperando contribuir con un soporte morfológico a la clínica quirúrgica de estas importantes estructuras de la rodilla.

\section{MATERIAL Y MÉTODO}

Se estudiaron 30 miembros inferiores (16 derechos y 14 izquierdos), de individuos chilenos de sexo no conocido. Estas piezas se encontraban fijadas en una solución de formaldehído al $10 \%$ y todas pertenecían al Departamento de Anatomía de la Universidad Católica del Maule.

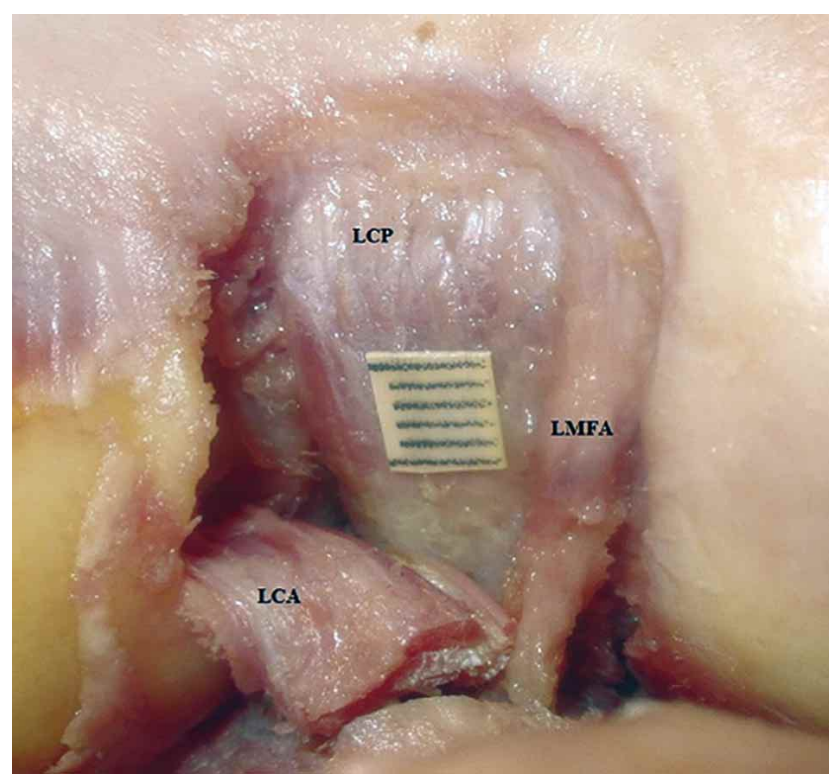

Fig. 1. Vista anterior de la articulación de la rodilla derecha. LMFA. Ligamento meniscofemoral anterior; LCP. Ligamento cruzado posterior; LCA. Ligamento cruzado anterior.
La disección mesoscópica se realizó con material quirúrgico y una lupa marca Ransor ${ }^{\circledR}$ (5X). Para la delimitación y disección de la región de la rodilla se prosiguió según técnica descrita por Testut \& Jacob.

Se determinó la frecuencia de las estructuras en estudio (LMFA y LMFP) y desde el punto de vista biométrico se efectuaron las siguientes mediciones: longitud, el ancho en tres secciones (a nivel de sus inserciones proximal, distal $\mathrm{y}$ en su punto medio) y el espesor en la parte media.

Las medidas se expresaron en milímetros (mm) utilizando un cáliper electrónico digital, marca Mitutoyo ${ }^{\circledR}$ de precisión $0,01 \mathrm{~mm}$.

Una vez realizada la disección y registradas las medidas, las muestras fueron fotografiadas. Se realizó un análisis estadístico descriptivo con medidas de tendencia central como media, desviación estándar (DS) y pruebas de significancia estadística con programa GraphPad Prism versión 6.01 .

\section{RESULTADOS}

La frecuencia de los LMFA (Fig. 1) y LMFP (Fig. 2) en un total de 30 rodillas, 14 izquierdas $(46,7 \%$ ) y 16 derechas $(53,3 \%)$ se muestra en la Tabla I.

Para comparar la frecuencia del LMFP (70 \%), en relación al LMFA (43,3\%), se aplicó la prueba estadística de Chi- Cuadrado con un nivel de significación de alpha de 0,05 y un valor $p$ de 0,04 lo que es estadísticamente significativo.

Los parámetros biométricos son presentados en las Tablas II y III. La comparación de los parámetros biométricos de los ligamentos meniscofemorales se realizó por medio de la Prueba t no pareada con la corrección de Welch (nivel de significancia de alpha de 0,05 ) obteniéndose los siguientes resultados:

Longitud promedio, valor $\mathrm{p}$ de 0,12 no estadísticamente significativo.

Tabla I. Frecuencia de los ligamentos meniscofemorales.

\begin{tabular}{lcccccccc}
\hline \multirow{2}{*}{ Rodillas } & \multicolumn{2}{c}{ Al menos un LMF } & \multicolumn{2}{c}{ Sólo LMFA } & \multicolumn{2}{c}{ Sólo LMFP } & \multicolumn{2}{c}{ Ambos } \\
\cline { 2 - 9 } & $\mathbf{n}$ & $\mathbf{\%}$ & $\mathbf{n}$ & $\mathbf{\%}$ & $\mathbf{n}$ & $\mathbf{\%}$ & $\mathbf{n}$ & $\mathbf{\%}$ \\
\hline Derecha & 14 & 46,7 & 3 & 10,0 & 7 & 23,3 & 4 & 13,3 \\
Izquier da & 12 & 40,0 & 2 & 6,7 & 6 & 20,0 & 4 & 13,4 \\
Total & 26 & 86,7 & 5 & 16,7 & 13 & 43,3 & 8 & 26,7 \\
\hline
\end{tabular}


- Ancho proximal promedio, valor p de 0,02 estadísticamente significativo.

- Ancho medio promedio, valor p de 0,16 no estadísticamente significativo.

-Ancho distal promedio, valor p de 0,001 estadísticamente significativo.

- Espesor medio promedio, valor p de 0,004 estadísticamente significativo.

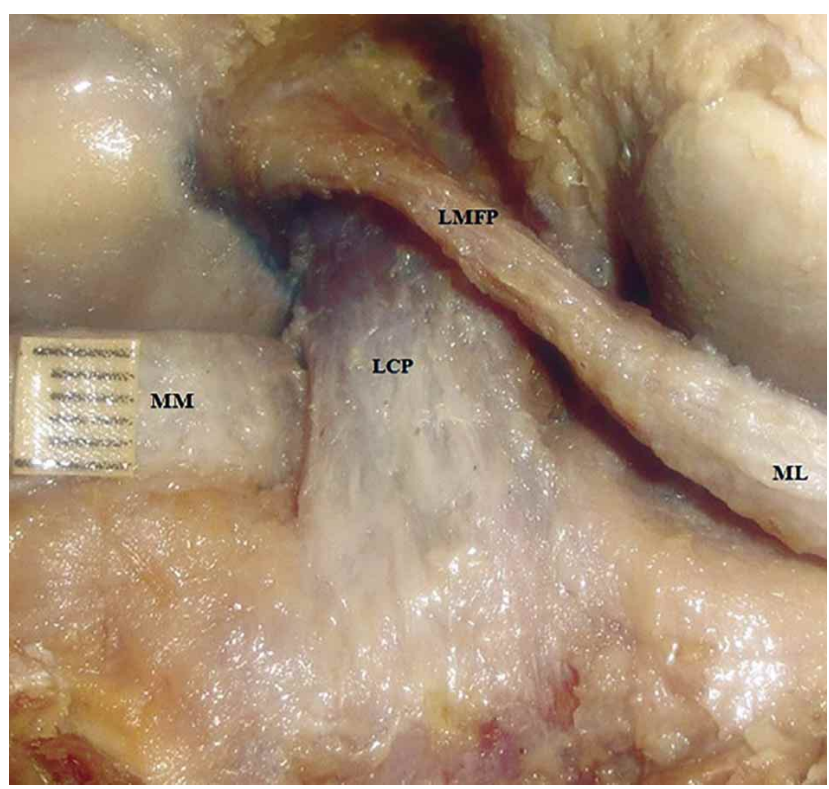

Fig. 2. Vista posterior de la articulación de la rodilla derecha. LMFP. Ligamento meniscofemoral posterior; LCP. Ligamento cruzado posterior; MM. Menisco medial; ML. Menisco lateral.

Tabla II. Registros de promedios y Desviación Estándar del LMFA en 13 Rodillas (en milímetros).

\begin{tabular}{lcccc}
\hline Parámetros & Promedio & DE & Mínimo & Máximo \\
\hline Longitud & 29,04 & $\pm 2,66$ & 23,93 & 33,25 \\
Ancho proximal & 9,88 & $\pm 4,34$ & 4,31 & 18,20 \\
Ancho medio & 4,95 & $\pm 1,98$ & 2,50 & 9,49 \\
Ancho distal & 5,84 & $\pm 1,95$ & 2,44 & 8,91 \\
Espesor medio & 1,19 & $\pm 0,47$ & 0,53 & 2,20 \\
\hline
\end{tabular}

Tabla III. Registros de promedios y Desviación Estándar del LMFP en 21 Rodillas (en milímetros).

\begin{tabular}{lcccc}
\hline Parámetros & Promedio & DE & Mínimo & Máximo \\
\hline Longitud & 27,46 & $\pm 3,08$ & 23,25 & 33,90 \\
Ancho proximal & 6,55 & $\pm 2,14$ & 3,60 & 10,49 \\
Ancho medio & 4,06 & $\pm 1,13$ & 2,11 & 6,70 \\
Ancho distal & 3,06 & $\pm 0,65$ & 2,36 & 4,42 \\
Espesor medio & 1,80 & $\pm 0,68$ & 0,86 & 2,96
\end{tabular}

\section{DISCUSIÓN}

Los LMF son considerados refuerzos del LCP, éstos se originan del asta posterior del menisco lateral y se dirigen al cóndilo medial del fémur. Actualmente los tratados clásicos carecen de una descripción detallada de estos ligamentos, algunos autores señalaron sólo al LMFP (Poirier \& Charpy; Orts Llorca; Bouchet \& Cuilleret; Latarjet et al.; Moore et $a l$.), e incluso otros no especifican si el recorrido del ligamento es anterior o posterior al LCP (Gray, 1893; Gegenbaur, 1899; Balli et al., 1932; Pernkopf et al., 1955.; Testut \& Jacob; García-Porrero \& Hurlé). Kapandji (1998) describió a estos ligamentos como parte de las bandas que conforman al LCP.

Autores más antiguos como Jamain (1862) y Chiarugi (1926) señalaron que se originan del LCP hacia el menisco lateral, en circunstancias que lo observado en este trabajo es que su origen es en el menisco lateral, tal cual lo han señalado autores clásicos como Testut \& Jacob; Testut \& Latarjet; Chatain \& Bustamante \& Rouvière et al.

La frecuencia de estas estructuras también es variable, Breathnach (1958); Testut \& Latarjet; Chatain \& Bustamante; Williams y Rouvière et al. indicaron que en ocasiones pueden encontrarse ambos o sólo uno. Nuestros hallazgos señalan que al menos uno de los LMF se visualizó en la mayoría de los casos y ambos en alrededor de un tercio, similar a lo reportado por Candiolo \& Guatero; Wan \& Felle y Gupte et al. (2002b), e incluso en algunos estudios la presencia de al menos uno fue en un 100\% de los casos (Hassine et al., 1992; Kusuyama et al., 1994; Poynton et al.; Ranalleta et al.; Amadi et al., 2008), lo que demuestra una alta presencia de estas estructuras.

En el presente estudio se observó que la presencia del LMFP es más constante que la del LMFA $70 \%$ y 43,3\%, respectivamente, siendo esta diferencia estadísticamente significativa (prueba de Chi cuadrado, valor $\mathrm{p}=0,04$ ). El porcentaje del LMFP es similar a lo descrito por Gupte et al. (2003) que de un total de 808 rodillas lo registraron en el 70,2\% de los casos y el LMFA estuvo presente en un $54 \%$.

La frecuencia del LMFP es similar a lo reportado por Kusuyama et al. (77 \%). En relación al LMFA existe concordancia con Candiolo \& Guatero (50 \%) y Wan \& Felle (33 $\%)$. Estos mismos autores describieron una mayor presencia del LMFP sobre el LMFA (Candiolo \& Guatero, LMFP $60 \%$ - LMFA 50 \%; Kusuyama et al. LMFP 77 \% -LMFA 69 \%; Wan \& Felle, LMFP 93 \% - LMFA $33 \%$ ), además Poynton et al., describieron un $90 \%$ para el LMFP y un $33 \%$ para el LMFA, y Gupte et al. (2002b) un $68 \%$ para el LMFP y para el LMFA un $64 \%$, tendencia que concuerda con nuestros registros. Llama la atención que en los dos estudios de raza asiáti- 
ca Cho et al. (1999) y Han et al., sus resultados muestren tendencia similar con una mayor presencia del LMFP (LMFP $89 \%$-LMFA $0 \%$ y LMFP $87 \%$-LMFA 1,9 \%, respectivamente), pero con una escasa frecuencia e incluso nula del LMFA, lo que se puede atribuir a la diferencias de raza pues en todos los estudios europeos y americanos el LMFA se detectó en aproximadamente un tercio de las muestras.

Sin embargo, nuestros valores difieren a lo investigado por Heller \& Langman, quienes sostuvieron que existe una leve diferencia en la frecuencia de estos ligamentos (LMFA $36 \%$ - LMFP $35 \%$ ); Hassine et al., detectaron al LMFA en el $100 \%$ de los casos y al LMFP en el $82 \%$; Gupte et al. (2002a) identificaron el LMFA en el $74 \%$ y el LMFP en el $69 \%$ de las muestras; Ranalleta et al., observaron al LMFA en el $100 \%$ de las rodillas y al LMFP en el $70 \%$ y Amadi et al., visualizaron el LMFA en el $100 \%$ de las muestras y el LMFP en $80 \%$. Estos estudios han mostrado que la presencia de ambos ligamentos es frecuente, e inversamente, mayor presencia del LMFA.

En relación a los parámetros biométricos de los ligamentos, en el presente estudio se registró que la longitud promedio del LMFA $(29,04 \pm 2,66 \mathrm{~mm})$ es levemente mayor en comparación con la del LMFP $(27,46 \pm 3,08 \mathrm{~mm})$ siendo no estadísticamente significativa (prueba t no pareada con la corrección de Welch, valor $\mathrm{p}=0,12$ ), diferente de lo expresado en la literatura consultada.

Candiolo \& Guatero informaron que el LMFP tuvo una longitud de 23,4 mm y el LMFA de 21,6 mm; Gupte et al. (2002a) indicaron para el LMFA un largo de 20,7 $\pm 3,9 \mathrm{~mm}$ y para el LMFP $23 \pm 4,2 \mathrm{~mm}$. Por otra parte Poynton et al., reportaron este parámetro según sexo, la longitud del LMFA en las muestras masculinas fue de 27,09 $\pm 2,15 \mathrm{~mm}$ y en las femeninas de 24,38 $\pm 3,39 \mathrm{~mm}$, estos datos son menores que en el LMFP (muestras masculinas 31,3 $\pm 2,54 \mathrm{~mm}$ y femeninas 27,59 $\pm 3,74 \mathrm{~mm})$. Erbagci et al., por medio de RM también describieron la longitud de los ligamentos según sexo, donde las rodillas femeninas tuvieron para el LMFA una longitud promedio de $9,87 \pm 4,79 \mathrm{~mm}$ y las masculinas $11,11 \pm 2,57 \mathrm{~mm}$; el LMFP midió 25,60 $\pm 5,50 \mathrm{~mm}$ en el sexo femenino y $28,80 \pm 5,49 \mathrm{~mm}$ en el masculino. En este último estudio se observó que la longitud del LMFA es muy inferior en relación al LMFP, pudiendo ser el resultado de un examen por imagen lo que podría haber dificultado la individualización del mismo, ya que podría haber estado adherido al LCP.

En la presente investigación, el ancho distal (origen

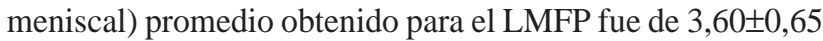
$\mathrm{mm}$, el cual es inferior en comparación al del LMFA cuyo

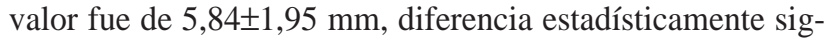
nificativa (prueba t no pareada con la corrección de Welch, valor $\mathrm{p}=0,001)$ Este registro concuerda con lo reportado por Candiolo \& Guatero, donde el ancho de origen para el LMFP fue de 4,3 mm y para el LMFA de 5,8 mm, siendo éste último valor, similar a lo descrito en esta investigación.

Con respecto al ancho proximal (inserción condilar) promedio se determinó un valor menor para el LMFP en rela-

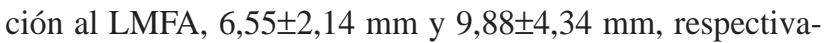
mente, esta diferencia es estadísticamente significativa (prueba t no pareada con la corrección de Welch, valor $\mathrm{p}=0,02$ ). Esto contrasta con lo informado por Candiolo \& Guatero donde el ancho de inserción condilar del LMFP ( $9,8 \mathrm{~mm}$ ) es superior al del LMFA $(8,6 \mathrm{~mm})$. La variable ancho medio promedio de nuestro estudio para el LMFA fue de 4,95 $\pm 1,98 \mathrm{~mm}$ registro superior al del LMFP de 4,06 $\pm 1,13 \mathrm{~mm}$, lo que no es estadísticamente significativo (prueba t no pareada con la corrección de Welch, valor $\mathrm{p}=0,16$ ) diferente de lo descrito por Poynton et al., tanto en las muestras de sexo masculino (LMFA $5,09 \pm 1,41 \mathrm{~mm}$ y LMFP 5,48 $\pm 2,13 \mathrm{~mm}$ ) como en las de sexo femenino (LMFA 2,99 $\pm 1,29 \mathrm{~mm}$ y LMFP 3,79 $\pm 2,56 \mathrm{~mm}$ ).

Erbagci et al., indicaron un ancho medio promedio en las muestras femeninas para el LMFA de 2,45 $\pm 1,02 \mathrm{~mm}$ y para el LMFP de 2,30 $\pm 1,15 \mathrm{~mm}$; en las masculinas el ancho del LMFA fue de 2,52 $\pm 0,87 \mathrm{~mm}$ y para el LMFP de 2,30 $\pm 1,15$ $\mathrm{mm}$, valores menores a nuestros registros. A pesar de éstos ser menores que los de nuestra serie, la tendencia es que el LMFA es mayor que el posterior, que podría atribuirse al escaso número de muestras que presentaron el LMFA.

En relación al espesor medio del LMFP se registró un

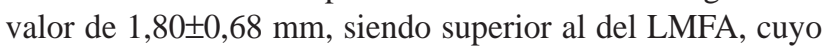
promedio fue de $1,19 \pm 0,47 \mathrm{~mm}$, esta diferencia es estadísticamente significativa (prueba t no pareada con la corrección de Welch, valor $\mathrm{p}=0,004$ ), parámetro no encontrado en la literatura revisada.

Los resultados expuestos en la presente investigación sirven para comprobar la frecuencia de estas estructuras en individuos de nuestra población y demostrar que la presencia de éstos no es menor. Además, los parámetros estudiados complementarán el conocimiento de estos ligamentos, aportando datos morfológicos y biométricos a la anatomía clínica y quirúrgica de la rodilla.

\section{AGRADECIMIENTOS}

A la Profesora Mg. Sra. Aglae Soto P., Profesora de Anatomía Humana de la Facultad de Medicina, Universidad Católica del Maule, Talca, por su disposición y gentileza, autorizando el uso de las muestras utilizadas en esta investigación. 
VILLARROEL, G. \& OLAVE, E. Biometrical characteristics of meniscofemoral ligaments in Chilean individuals. Int. J. Morphol., 34(3):860-865, 2016.

SUMMARY: The knowledge of the meniscofemoral ligaments (MFL) is of great importance to the anatomy and surgical clinic. In order to conduct an analysis of their frequency and biometry in Chilean individuals, 30 knees of corpses of adult individuals, sex unknown, fixed in $10 \%$ formaldehyde and belonging to the Laboratory of Anatomy, Faculty of Medicine of the Universidad Católica del Maule, Chile. Using a digital caliper, measurements of length, width and the thickness to different levels were recorded. All the samples studied were photographed. Regarding frequency, PMFL occurred in 21 cases (70 \%) and AMFL in 13 (43.4 \%). The average length of AMFL was $29.04 \pm 2.66 \mathrm{~mm}$ and the PMFL $27.46 \pm 3.08 \mathrm{~mm}$; the proximal width of the AMFL was $9.88 \pm 4.34 \mathrm{~mm}$ and the PMFL of $6.55 \pm 2.14 \mathrm{~mm}$ and the average thickness of the AMFL was $1.19 \pm 0.47 \mathrm{~mm}$ and the PMFL of $1.8 \pm 0.68 \mathrm{~mm}$. Other measurements are shown in the text. The obtained results are a contribution to the knowledge of ligaments of the knee joint in our population.

KEY WORDS: Anatomy; Knee; Anterior meniscofemoral ligament; Posterior meniscofemoral ligament; Posterior cruciate ligament.

\section{REFERENCIAS BIBLIOGRÁFICAS}

Amadi, H. O.; Gupte, C. M.; Lie, D. T.; McDermott, I. D.; Amis, A. A. \& Bull, A. M. A biomechanical study of the meniscofemoral ligaments and their contribution to contact pressure reduction in the knee. Knee Surg. Sports Traumatol. Arthrosc., 16(11):1004-8, 2008.

Balli, R.; Bertelli, D.; Bruni, A. C.; Giannelli, L.; Luna, E.; Pende, N.; Sala, L.; Salvi, G. \& Versari R. Trattato di Anatomia Umana. Volumen I: Osteologia e Sindesmologia. $2^{\text {a }}$ ed., Milano, Biblioteca Medica Italiana, Editore Dott. Francesco Vallardi, 1932. pp.3041.

Brantigan, O. C. \& Voshell, A. F. Ligaments of the knee joint; the relationship of the ligament of Humphry to the ligament of Wrisberg. J. Bone Joint Surg. Am., 28:66, 1946.

Breathnach, A. S. Frazer's Anatomy of the Human Skeleton. 5a ed. London, J. Churchill Ltd., 1958. pp.255.

Bouchet, A. \& Cuilleret, J. Anatomía descriptiva, topográfica y funcional. Miembros inferiores. Buenos Aires, Médica Panamericana, 1984. p.296.

Candiollo, L. \& Gautero, G. Morphologie et fonction des ligaments ménisco-fémoraux de l'articulation du genou chez l'homme. Acta Anat., 38:304-23, 1959.

Chatain, L. I. \& Bustamante, B. J. Anatomía Macroscópica Funcional y Clínica. México D. F., Addison-Wesley Iberoamericana, 1986. p.818.

Chiarugi, G. Istituzioni di Anatomia dell'Uomo. Volume Primo. Introduzione - Anatomia Generale - Embriogenia - Anatomia Sistematica: Apparecchio Tegumentario - Apparecchio Scheletrico. Milano, Società Editrice Libraria, 1926. pp.836.

Cho, J. M.; Suh, J. S.; Na, J. B.; Cho, J. H.; Kim, Y.; Yoo, W. K.; Lee, H. Y. \& Chung, I. H. Variations in meniscofemoral ligaments at anatomical study and MR imaging. Skeletal Radiol., 28(4):189-95, 1999.

Erbagci, H.; Yildirim, H.; Kizilkan, N. \& Gümüsburum E. An MRI study of the meniscofemoral and transverse ligaments of the knee. Surg. Radiol. Anat., 24(2):120-4, 2002.

García-Porrero, J. A. \& Hurlé, J. M. Anatomía Humana. Madrid, McGraw-Hill/Interamericana, 2005. p.991.

Gegenbaur, C. Lehrbuch der Anatomie des Menschen. $7^{\text {a }}$ ed. Leipzig, W. Engelmann, 1899. p.486.

Gray, H. Anatomy, Descriptive and Surgical. 13 a ed., Philadelphia, Lea Brothers \& Co., 1893. p.1125.

Gupte, C. M.; Smith, A.; McDermontt, I. D.; Bull, A. M.; Thomas, R. D. \& Amis, A. A. Meniscofemoral ligaments revisited. Anatomical study, age correlation and clinical implications. J. Bone Joint Surg. Br., 84(6):846-51, 2002a.

Gupte, C. M.; Smith, A.; Jamieson, N.; Bull, A. M.; Thomas, R. D. \& Amis, A. A. Meniscofemoral ligaments--structural and material properties. J. Biomech., 35(12):1623-9, 2002 b.

Gupte, C. M.; Bull, A. M.; Thomas, R. D. \& Amis, A. A. A review of the function and biomechanics of the meniscofemoral ligaments. Arthroscopy, 19(2):161-71, 2003.

Han, S. H.; Kim, D. I.; Choi, S. G.; Lee, J. H. \& Kim, Y. S. The posterior meniscofemoral ligament: morphologic study and anatomic classification. Clin. Anat., 25(5):634-40, 2012.

Hassine, D.; Feron, J. M.; Henry-Feugeas, M. C.; SchoumanClaeys, E.; Guérin Surville, H. \& Frija, G. The meniscofemoral ligaments: magnetic resonance imaging and anatomic correlations. Surg. Radiol. Anat., 14(1):59-63, 1992. 
Heller, L. \& Langman, J. The menisco-femoral ligaments of the human knee. J. Bone Joint Surg. Br., 46(2):307-13, 1964.

Jamain, A. Nuevo Tratado Elemental de Anatomía Descriptiva y de Preparaciones Anatómicas. $2^{\mathrm{a}}$ ed. Madrid, Carlos BaillyBailliere, 1862. pp.911.

Kapandji, A. I. Fisiología Articular. Tomo 2. Miembro Inferior. $5^{\text {a }}$ ed., Madrid, Médica Panamericana, 1998.

Kohn, D. \& Moreno, B. Meniscus insertion anatomy as a basis for meniscus replacement: a morphological cadaveric study. Arthroscopy, 11(1):96-103, 1995.

Kusayama, T.; Harner, C. D.; Carlin, G. J.; Xerogeanes, J. W. \& Smith, B. A. Anatomical and biomechanical characteristics of human meniscofemoral ligaments. Knee Surg. Sports Traumatol. Arthrosc., 2(4):234-7, 1994.

Latarjet, M.; Ruiz Liard, A. \& Pró, E. A. Anatomía Humana. Tomo I. $4^{\text {a }}$ ed. Buenos Aires, Médica Panamericana, 2011. p.928.

Moore, K. L.; Dalley, A. F. \& Agur, A. M. R. Anatomía con Orientación Clínica. $6^{\mathrm{a}}$ ed., Barcelona, Lippincot Williams \& Wilkins, 2010. p.1162.

Orts Llorca, F. Anatomía Humana. Tomo Primero. $3^{\mathrm{a}}$ ed. Barcelona, Científico-Médica, 1964. p.869.

Pernkopf, E. Anatomía Topográfica Humana: Texto y Atlas para la Disección por Regiones y Planos. Tomo 2. Abdomen, Pelvis y Extremidades Abdominales. 2a ed. Barcelona, Labor, 1955.

Poirier, P. \& Charpy, A. Traité d'Anatomie Humaine. Tome Premier, Embryologie, Osteologie, Arthrologie. $2^{\mathrm{a}}$ ed., Paris, Masson, 1899. p.724.

Poynton, A. R.; Javadpour, S. M.; Finegan, P. J. \& O’Brien, M. The meniscofemoral ligaments of the knee. J. Bone Joint Surg. Br., 79(2):327-30, 1997.

Ranalletta, M.; Rossi, W.; Brigatti, N. \& Ranalletta, A. Estudio anatómico de los ligamentos menisco femorales de la rodi1la. Rev. Argent. Artrosc., 11(1):51-3, 2004.

Rouvière, H.; Delmas, A. \& Delmas, V. Anatomía Humana Descriptiva, Topográfica y Funcional. Tomo 3: Miembros. 11 ed. Barcelona, Masson, 2005. p.668 .

Testut, L. \& Jacob, O. Tratado de Anatomía Topográfica: con Aplicaciones Medicoquirúrgicas. Tomo Segundo: AbdomenPelvis-Miembros. $8^{a}$ ed. Barcelona, Salvat, 1961. p.889.

Testut, L. \& Latarjet, A. Tratado de Anatomía Humana. Tomo Primero: Osteología- Artrología-Miología. 9a ed. Barcelona, Salvat, 1969. p.1213.
Wan, A. C. \& Felle, P. The menisco-femoral ligaments. Clin. Anat., 8(5):323-6, 1995.

Williams, P. L. Anatomía de Gray: Bases Anatómicas de la Medicina y Cirugía. Tomo I. 38 ed. Madrid, Harcourt Brace, 2001. p.900.

Dirección para Correspondencia:

Dr. Enrique Olave

Facultad de Medicina

Universidad de La Frontera

Temuco

CHILE

Email: enrique.olave@ufrontera.cl

Recibido : 07-04-2016

Aceptado: 25-07-2016 